\title{
Development of Primary Thyroid Lymphoma during an Ultrasonographic Follow-up of Hashimoto's Thyroiditis: A Report of 9 Cases
}

\author{
Tetsuya Mizokami ${ }^{1}$, Katsuhiko Hamada ${ }^{1}$, Tetsushi Maruta ${ }^{1}$, Kiichiro Higashi ${ }^{1}$, \\ Hiroto Yamashita ${ }^{2}$, Yasushi Noguchi ${ }^{2}$, Hitoshi Noguchi ${ }^{2}$ and Junichi Tajiri ${ }^{1}$
}

\begin{abstract}
We herein experienced 9 patients with primary thyroid lymphoma that developed during 3-18 years of ultrasonographic follow-up of Hashimoto's thyroiditis. All nine patients had localized mucosa-associated lymphoid tissue (MALT) lymphoma. Two patients had diffuse type, one had mixed type, and six had nodular type according to the ultrasonographic classification. A clearly enlarging goiter was observed before the diagnosis of lymphoma in 3 patients. An enlarging goiter was not apparent in the remaining 6 patients with nodular type lymphoma, however, the emergence or enlargement of a hypoechoic nodular lesion was observed. Thyroid MALT lymphoma may be diagnosed early by an ultrasonographic follow-up of Hashimoto's thyroiditis.
\end{abstract}

Key words: thyroid lymphoma, MALT lymphoma, Hashimoto's thyroiditis, ultrasonography

(Intern Med 55: 943-948, 2016)

(DOI: 10.2169/internalmedicine.55.5428)

\section{Introduction}

Primary thyroid lymphoma accounts for $1-5 \%$ of all thyroid malignancies and has been estimated to account for 1$2 \%$ of all extranodal lymphomas (1). Primary thyroid lymphomas are presumed to develop from lymphoid tissue acquired during the course of an autoimmune process (2). The prevalence of Hashimoto's thyroiditis in patients with primary thyroid lymphoma is nearly $100 \%$ according to thorough histopathological examinations (3). The risk of patients with Hashimoto's thyroiditis developing primary thyroid lymphoma is 40 to 80 times greater than that of the general population (4).

The most classical presentation of primary thyroid lymphoma is a rapidly enlarging goiter in patients with preexisting Hashimoto's thyroiditis. However, it is not uncommon for primary thyroid lymphoma to grow gradually for several months to several years $(4,5)$. There are few reports on the serial ultrasonographic changes with the development of primary thyroid lymphoma in a background of Hashimoto's thyroiditis (5). We herein report 9 patients with primary thyroid lymphoma which developed during 3-18 years of ultrasonographic follow-up of Hashimoto's thyroiditis.

The diagnosis of thyroid lymphoma was made histopathologically using tissues obtained by an open biopsy or thyroidectomy in all subjects. A flow cytometric analysis was used auxiliary to establish monoclonality for the definitive diagnosis of lymphoma in some patients. When the result of the flow cytometric analysis was equivocal, a G-band analysis (karyotyping) and genetic analysis (immunoglobulin heavy chain gene rearrangements) were applied additionally to confirm monoclonality. Surgical procedures to remove a lesion or to obtain a diagnostic sample were determined by each surgeon. Lobectomy or partial thyroidectomy was performed to obtain enough tissue for a histopathological examination and flow cytometric analysis when the lesion was relatively small. Soon after the histopathological diagnosis of thyroid lymphoma, radiotherapy was indicated as primary treatment for patients with stage IE or IIE disease.

Patients underwent an ultrasonographic examination mostly at 6 to 12 -month intervals. The intervals of the ultra-

${ }^{1}$ Tajiri Thyroid Clinic, Japan and ${ }^{2}$ Noguchi Thyroid Clinic and Hospital Foundation, Japan

Received for publication March 23, 2015; Accepted for publication July 27, 2015

Correspondence to Dr. Tetsuya Mizokami, tetsuyamizokami@hotmail.com 
Table 1. Clinical Characteristics of the Cases.

\begin{tabular}{|c|c|c|c|c|c|c|c|c|c|c|}
\hline \multirow[t]{2}{*}{ Case } & \multirow{2}{*}{$\begin{array}{c}\text { Age } \\
\text { At Dx } \\
\text { (First visit) }\end{array}$} & \multicolumn{3}{|c|}{ Thyroid function at Dx } & \multicolumn{2}{|c|}{ Thyroid autoantibodies } & \multirow{2}{*}{$\begin{array}{c}\text { Echo pattern } \\
\text { of } \\
\text { lymphoma }\end{array}$} & \multicolumn{3}{|c|}{ Thyroid weight } \\
\hline & & $\begin{array}{c}\text { FT4 } \\
(\mathrm{ng} / \mathrm{dL})\end{array}$ & $\begin{array}{c}\text { TSH } \\
(\mu \mathrm{IU} / \mathrm{mL})\end{array}$ & $\begin{array}{c}\text { LT4 } \\
(\mu \mathrm{g} / \text { day })\end{array}$ & $\begin{array}{c}\text { MCPA } \\
\text { (anti-TPO) }\end{array}$ & $\begin{array}{c}\text { TGPA } \\
\text { (anti-Tg) }\end{array}$ & & $\begin{array}{c}\text { At Dx } \\
(\mathrm{g})\end{array}$ & $\begin{array}{r}\text { Las } \\
(\mathrm{g} ; \mathrm{p} \\
\end{array}$ & $\begin{array}{l}\text { visit } \\
\text { eriod) }\end{array}$ \\
\hline 1 & $70(67)$ & 1.76 & 0.02 & 100 & $\times 102,400$ & $\times 102,400$ & diffuse & 86 & 66 & $-6 m$ \\
\hline 2 & $66(63)$ & 1.82 & 0.24 & 0 & $\times 1,600$ & n.d. & diffuse & 46 & 31 & $-3 m$ \\
\hline 3 & $67(60)$ & 1.47 & 2.87 & 75 & $\times 102,400$ & n.d. & mixed & 60 & 40 & $-12 \mathrm{~m}$ \\
\hline 4 & $67(62)$ & 1.11 & 1.09 & 50 & $(127 \mathrm{IU} / \mathrm{mL})$ & $(534 \mathrm{IU} / \mathrm{mL})$ & nodular & 51 & 46 & $-6 m$ \\
\hline 5 & $53(47)$ & 1.35 & 3.60 & 100 & $\times 100$ & n.d. & nodular & 26 & 25 & $-6 m$ \\
\hline 6 & $75(65)$ & 1.07 & 2.21 & 25 & n.d. & $\times 400$ & nodular & 17 & 16 & $-5 \mathrm{~m}$ \\
\hline 7 & $73(55)$ & 1.20 & 1.77 & 50 & $\times 102,400$ & $(4,480 \mathrm{IU} / \mathrm{mL})$ & nodular & 46 & 43 & $-2 \mathrm{~m}$ \\
\hline 8 & $85(78)$ & 1.01 & 5.40 & 0 & $\times 102,400$ & $\times 1,600$ & nodular & 29 & 25 & $-11 \mathrm{~m}$ \\
\hline 9 & $85(77)$ & 1.30 & 2.93 & 50 & n.d. & $\times 400$ & nodular & 42 & 42 & $-7 \mathrm{~m}$ \\
\hline
\end{tabular}

At Dx: at diagnosis of primary thyroid lymphoma,

FT4: serum free thyroxine level, TSH: serum thyrotropin level, LT4: replacement dose of L-thyroxine,

MCPA: anti-thyroid microsomal particle agglutination, TGPA: anti-thyroglobulin particle agglutination,

anti-Tg: anti-thyroglobulin antibody, anti-TPO: anti-thyroid peroxidase antibody, n.d.: not detected

sonographic examination were irregularly shortened to a few months for a careful follow-up of some noticeable findings. The thyroid weight was estimated by ultrasound as previously reported (6). Ultrasonographic patterns of the primary thyroid lymphoma were classified into three types according to internal echoes, border, and posterior echoes: diffuse, mixed, and nodular types according to the classification proposed by Ota et al. (7).

Autoantibodies to thyroid microsomal antigen and thyroglobulin were measured by particle agglutination using commercial kits (Serodia-AMC and Serodia-ATG, Fujirebio Inc., Tokyo, Japan; normal values $<100 x$ and $<100 x$, respectively). In some patients, serum anti-thyroid peroxidase antibody and anti-thyroglobulin antibody were measured by commercial electrochemiluminescence immunoassays (Elecsys Anti-TPO and Elecsys Anti-Tg; Roche Diagnostics, Tokyo, Japan; normal values $<16 \mathrm{IU} / \mathrm{mL}$ and $<28 \mathrm{IU} / \mathrm{mL}$, respectively). Serum levels of free thyroxine (T4) and thyrotropin (TSH) were measured by commercial immunoassays (Roche Diagnostics).

\section{Case Reports}

We experienced 9 patients with primary thyroid lymphoma between September 2005 and January 2014 who had been followed for Hashimoto's thyroiditis for at least 3 years and had at least one ultrasonogram within 12 months prior to their histological diagnosis of lymphoma. The patients' characteristics are listed in Tables 1 and 2. All 9 patients were women and had localized mucosa-associated lymphoid tissue (MALT) lymphoma. Seven patients had stage IE, and two (Cases 2 and 5) patients had stage IIE. ${ }^{67} \mathrm{Ga}$ whole-body scintigraphy was performed at the diagnosis of lymphoma in all patients. ${ }^{18} \mathrm{~F}$-fluorodeoxyglucose positron emission tomography/computed tomography was performed additionally to evaluate the stage shortly after the histological diagnosis of lymphoma in one patient, who demonstrated a weak uptake of ${ }^{67} \mathrm{Ga}$ in the thyroid before surgery (Case 7).
The age at diagnosis of lymphoma ranged from 53 to 85 years with a median age of 70 years, and the duration of follow-up ranged from 3 to 18 years. Seven patients had been treated for hypothyroidism due to Hashimoto's thyroiditis with LT4 (25-100 $\mu \mathrm{g}$ /day), however, the dosage of LT4 had not changed for several years before the diagnosis of lymphoma in any of the patients. All 9 patients were diagnosed with Hashimoto's thyroiditis according to a diffuse goiter with positivity for anti-thyroid autoantibodies. Regarding the ultrasonographic pattern, two patients had diffuse type lymphoma, one had mixed type, and 6 had nodular type (Fig. 1, 2).

A clearly enlarging goiter without significant change in the serum TSH level was observed within 12 months prior to the diagnosis of lymphoma in 3 patients (Cases 1-3) (Fig. 1, 3). Case 1 had a symmetric enlarging goiter, which was subjectively noticed one week before the diagnosis of thyroid lymphoma. The goiter was transiently enlarged 15 months before the diagnosis of lymphoma, however, the cytological diagnosis was Hashimoto's thyroiditis at that time. Case 2 had an asymmetrically enlarging right lobe of the thyroid. Right cervical lymphadenopathy was detected at the diagnosis of lymphoma. Case 3 had a markedly enlarging hypoechoic nodular lesion in the right lobe toward the isthmus and enlarging patchy hypoechoic lesions in the left lobe.

An enlarging goiter was not apparent in the remaining 6 patients (Fig. 2, 4). The emergence of a hypoechoic nodular lesion, which had not been detected 6-11 months earlier, was observed in these 6 patients. Two patients were diagnosed with primary thyroid lymphoma soon after the detection of a hypoechoic nodular lesion (Cases 8 and 9). Although the total thyroid volume did not noticeably increase, the nodular lesions enlarged during the careful ultrasonographic follow-up until the histological diagnosis of lymphoma in the other 4 patients (Cases 4-7). They were diagnosed with lymphoma 2-30 months after the first detection of a hypoechoic nodular lesion. Bilateral lymphadenopathy was detected at the diagnosis of lymphoma in Case 5. 
Case 1. A
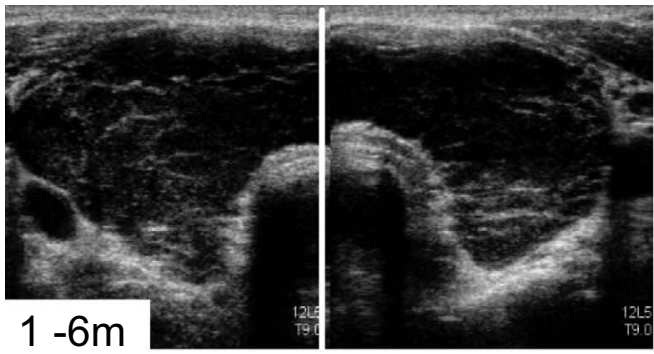

Case 2. A
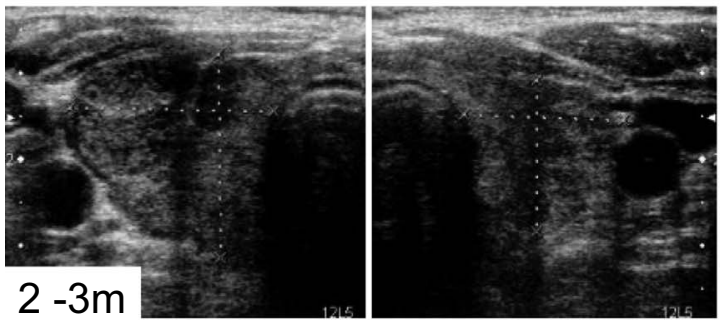

B
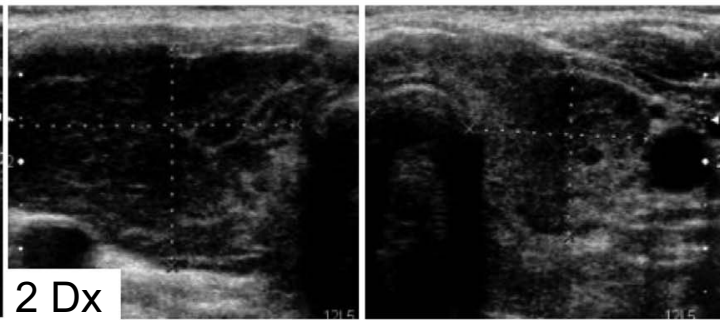

Case 3. A

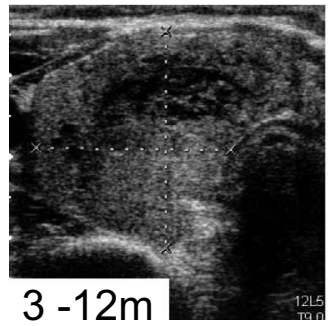

B

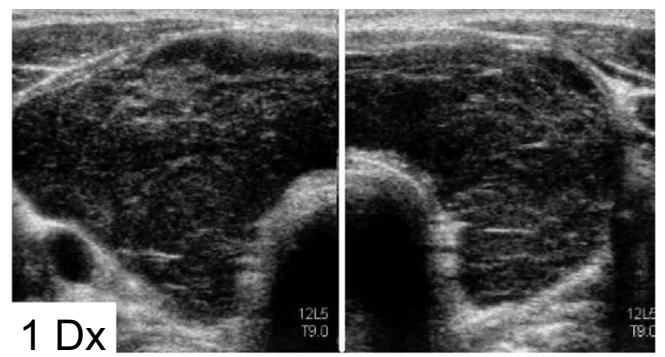

B

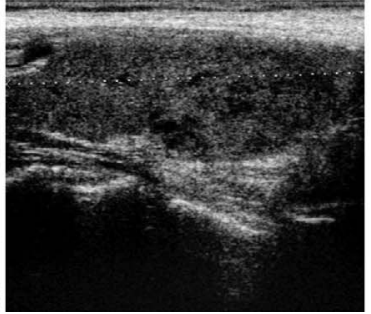

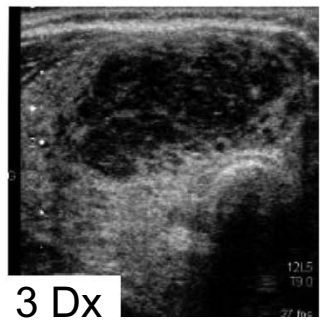

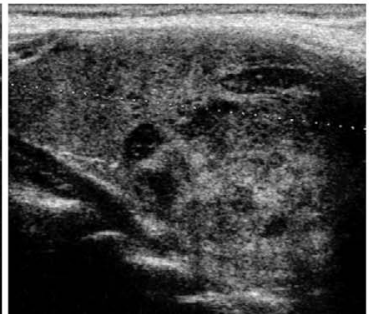

Figure 1. Ultrasonographic findings of cases with a clearly enlarging goiter. Case 1: A 70-year-old woman with primary thyroid lymphoma (mucosa-associated lymphoid tissue lymphoma; MALT lymphoma), diffuse type. Ultrasonographic images at 6 months earlier (A) and at diagnosis (B). A diffuse goiter had gradually enlarged over 12 months. Internal echoes were markedly hypoechoic, and the border between the lymphoma and nonlymphomatous tissues could not be identified. Case 2: A 66-year-old woman with primary thyroid lymphoma (MALT lymphoma), diffuse type. The ultrasonographic images at 3 months earlier (A) and at diagnosis (B). The right lobe had enlarged asymmetrically over 3 months. Internal echoes were markedly hypoechoic at diagnosis, and the border between the lymphoma and nonlymphomatous tissues could not be identified. Case 3: A 67-year-old woman with primary thyroid lymphoma (MALT lymphoma), mixed type. A hypoechoic nodular lesion was detected 12 months earlier (horizontal section) (A). The hypoechoic nodular lesion enlarged relatively rapidly toward the isthmus and became more hypoechoic or pseudocystic over 12 months (B). Patchy hypoechoic lesions in the left lobe had enlarged over 12 months (sagittal section).

Six patients (Cases 2-4, 6, 8, and 9) were strongly suspected to have lymphoma from the initial ultrasonographyguided fine-needle aspiration biopsy and soon underwent an open biopsy or thyroidectomy. On the other hand, the initial cytological diagnosis was Hashimoto's thyroiditis in 3 patients (Cases 1, 5, and 7). They underwent reexamination by a fine-needle aspiration biopsy due to an enlarging goiter, enlargement of a hypoechoic nodular lesion or development of cervical lymphadenopathy during 2-15 months of careful follow-up (Fig. 3, 4). Three patients (Cases 3-5) were diagnosed with primary thyroid lymphoma more than 11 months after the initial detection of a hypoechoic nodular lesion.
The histological diagnosis of lymphoma was made 30 months after the initial detection of a hypoechoic lesion in Case 4, which had gradually enlarged and showed pseudocystic change.

The treatment and clinical course of each patient are shown in Table 2. Six patients were treated with surgery followed by external irradiation, and one patient with external irradiation alone (open thyroid biopsy). Two patients with stage IE were treated with surgery alone (subtotal thyroidectomy with prophylactic cervical lymph node dissection). They did not undergo postsurgical radiotherapy because they refused radiotherapy due to advanced age. All patients, ex- 
Case 4. A
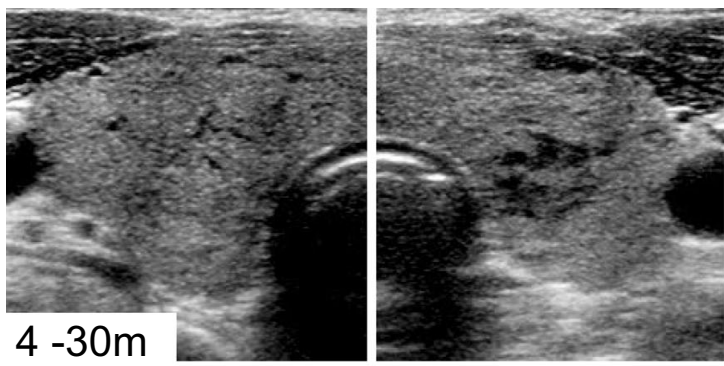

B
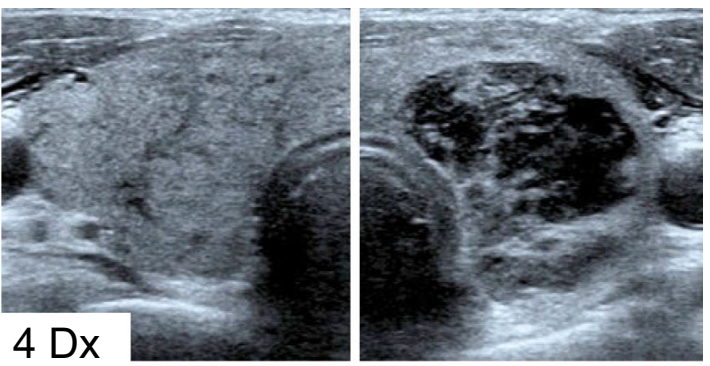

Case 5. A B

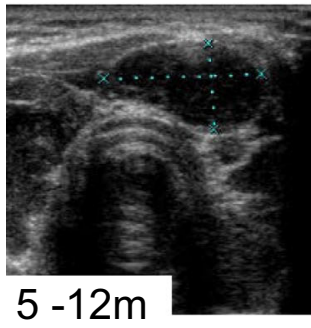

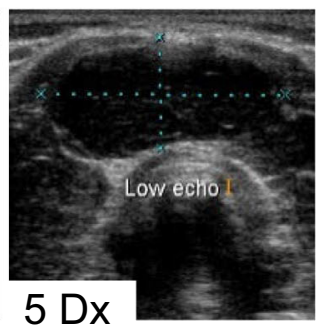

Case 6. A

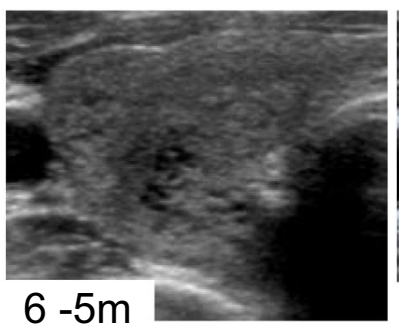

B

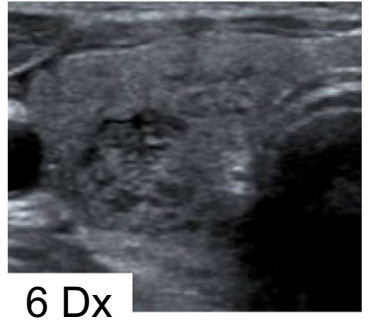

\section{Case 7. A}

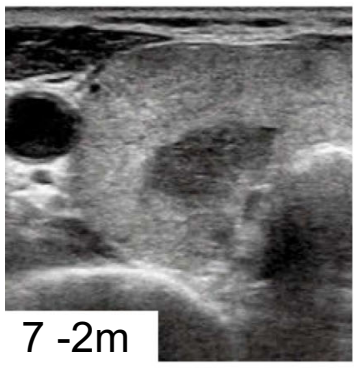

Case 8.

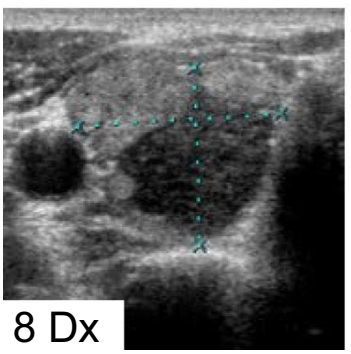

B
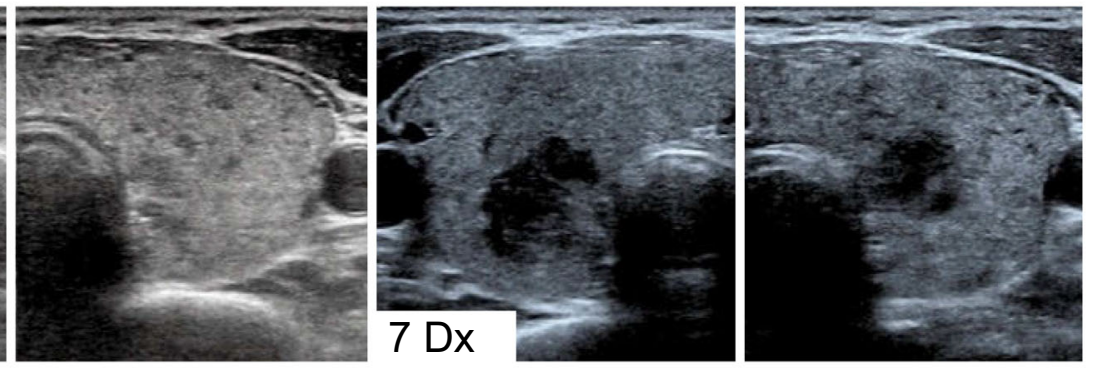

Figure 2. Ultrasonographic findings of cases without a clearly enlarging goiter. Case 4: A 67-yearold woman with primary thyroid lymphoma (MALT lymphoma), nodular type. Ultrasonographic images 30 months earlier (A) and at diagnosis (B). A pseudocystic nodular lesion had gradually enlarged over 30 months. Case 5: A 53-year-old woman with primary thyroid lymphoma (MALT lymphoma), nodular type. A pseudocystic nodular lesion was detected in the isthmus 12 months earlier (A) and had gradually enlarged over 12 months (B). Case 6: A 75-year-old woman with primary thyroid lymphoma (MALT lymphoma), nodular type. An enlarging hypoechoic lesion was detected in the right lobe 5 months earlier (A) and had enlarged over 5 months (B). Case 7: A 73-year-old woman with primary thyroid lymphoma (MALT lymphoma), nodular type. An enlarging hypoechoic and homogenous lesion was detected in the right lobe 2 months earlier (A). Moreover, another hypoechoic lesion was detected in the left lobe at diagnosis (B). Case 8: An 85-year-old woman with primary thyroid lymphoma (MALT lymphoma), nodular type. A marked hypoechoic nodular lesion had developed over 11 months. Case 9: An 85-year-old woman with primary thyroid lymphoma (MALT lymphoma), nodular type. A hypoechoic nodular lesion had developed over 7 months. 


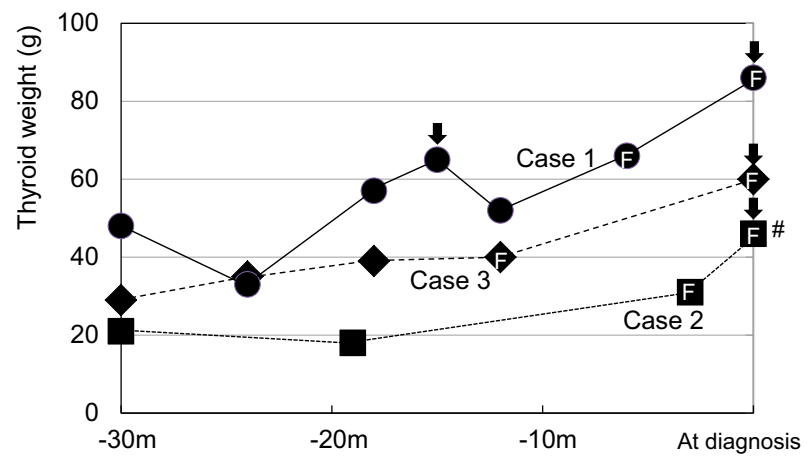

Figure 3. Estimated thyroid weights prior to the diagnosis of primary thyroid lymphoma. The thyroids at the diagnosis of lymphoma had obviously enlarged compared with the last measurement (3-12 months prior to the diagnosis of lymphoma). \#: cervical lymphadenopathy, F: see Fig. 1, arrows: an ultrasonography-guided fine-needle aspiration biopsy.

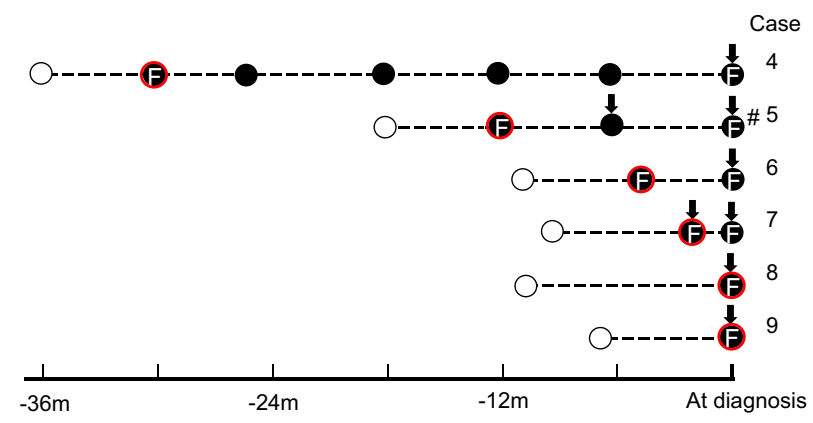

Figure 4. Detection of ultrasonographic lesions before the diagnosis of primary thyroid lymphoma. A hypoechoic nodular lesion, which had not been detected 6-11 months earlier, was observed (red circle). 0 : an ultrasonographic lesion of lymphoma. O: no ultrasonographic lesion of lymphoma. \#: cervical lymphadenopathy, F: see Fig. 2, arrows: an ultrasonographyguided fine-needle aspiration biopsy.

Table 2. Diagnosis and Treatment of the Cases.

\begin{tabular}{|c|c|c|c|c|c|}
\hline \multirow[t]{2}{*}{ Case } & \multicolumn{2}{|l|}{ Diagnosis } & \multirow[t]{2}{*}{ Treatment } & \multirow[t]{2}{*}{ Clinical course } & \multirow{2}{*}{$\begin{array}{l}\text { Follow-up } \\
\text { (yrs) }\end{array}$} \\
\hline & Operation or open biopsy & Ig light chain & & & \\
\hline 1 & Subtotal thyroidectomy & $\kappa$ chain & Surgery + irradiation (50 Gy) & Progression & 4 \\
\hline 2 & Right lobectomy & N.A. & Surgery + irradiation (50 Gy) & No recurrence & 8 \\
\hline 3 & Open thyroid biopsy & N.A. & Irradiation (50 Gy) & No recurrence & 7 \\
\hline 4 & Left lobectomy and right partial lobectomy & N.A. & Surgery + irradiation (40 Gy) & No recurrence & 2 \\
\hline 5 & Partial thyroidectomy (Isthmus) & N.A. & Surgery + irradiation (50 Gy) & No recurrence & 7 \\
\hline 6 & Right lobectomy and left partial lobectomy & Equivocal $^{*}$ & Surgery + irradiation (40 Gy) & No recurrence & 2 \\
\hline 7 & Left lobectomy and right partial lobectomy & $\lambda$ chain & Surgery + irradiation (30 Gy) & No recurrence & 1 \\
\hline 8 & Subtotal thyroidectomy & $\kappa$ chain & Surgery & No recurrence & 2 \\
\hline 9 & Subtotal thyroidectomy & N.A. & Surgery & No recurrence & 2 \\
\hline
\end{tabular}

*G-band analysis: 46, XX, t(11;17) (p15;q21), 46, XX, idem, add (9) (p13), IGH (JH) rearrangement: positive.

Ig light chain: immunoglobulin light chain expression by flow cytometry, Follow-up: period of follow-up after the initial treatment,

N.A.: not available

cept for one, had a good clinical course by surgery and/or radiotherapy. One patient (Case 1) was transferred to another hospital for chemotherapy since the lymphoma had spread to the tonsils and lungs during radiotherapy. All patients were alive during 1-8 years of follow-up after the initial treatment.

\section{Discussion}

Primary thyroid lymphoma is divided into two common histologic subtypes: MALT lymphomas vs. diffuse large Bcell lymphoma (DLBCL) and mixed MALT lymphoma/ DLBCL (4). MALT lymphomas account for 23 to $47 \%$ of primary thyroid lymphomas $(8,9)$. All 9 of the present patients had localized MALT lymphoma. Among the subtypes of primary thyroid lymphoma, MALT lymphoma is most strongly associated with Hashimoto's thyroiditis (10). It follows a relatively indolent clinical course and is more likely to present at an earlier stage and demonstrate a better response to treatment (11). However, MALT lymphoma may transform to a higher grade, more aggressive lymphoma, i.e., DLBCL or mixed MALT lymphoma/DLBCL (9). An ultrasonographic follow-up of Hashimoto's thyroiditis might facilitate the early detection of MALT lymphoma before transformation to DLBCL.

Regarding the ultrasonographic findings of lymphoma, 6 of the 9 present patients had nodular type lymphoma. The rates of each ultrasonographic type were previously reported to be as follows in 79 patients with primary thyroid lymphoma detected by ultrasonographic screening: nodular type $47 \%$, diffuse type $38 \%$, and mixed type $15 \%$ (7). The difference between the present patients and the above-reported pa- 
tients is that some lymphomas may have been detected earlier during the ultrasonographic follow-up for Hashimoto's thyroiditis in the present patients. Lymphoma was ultrasonographically classified as nodular type in all 6 of the present patients without an apparently enlarging goiter (Cases 4-9). Firstly, nodular type lymphoma may have higher ultrasonographic detectability than other types. Diffuse type lymphoma shows homogeneous and hypoechoic internal echoes, while Hashimoto's thyroiditis typically appears diffusely heterogeneous with hypoechogenicity on ultrasonography (12). However, it is occasionally difficult to differentiate diffuse type lymphoma from severe Hashimoto's thyroiditis by ultrasonography, making the early identification of diffuse type lymphoma difficult in patients with Hashimoto's thyroiditis. Secondly, some nodular type lymphomas might change to other types as the lymphoma grows.

Regarding the progression of primary thyroid lymphoma in 7 of the present patients (Cases 1-7), a clearly enlarging goiter was observed in 3 (Cases 1-3). The rates of progression differed among the individual patients, despite the same subtype of lymphoma, or MALT lymphoma. In 2 patients (Cases 4 and 5), the lymphoma gradually enlarged for more than 11 months after the initial detection of the hypoechoic nodular lesion. Recently, the proportion of patients with primary thyroid lymphoma who develop a rapidly enlarging goiter has decreased (7). One of the reasons for this finding is that smaller primary thyroid lymphoma has been detected by high-resolution ultrasonography and diagnosed with an ultrasonography-guided fine-needle aspiration biopsy.

Although the initial cytological diagnosis was Hashimoto's thyroiditis in 3 patients (Cases 1, 5, and 7), lymphoma was strongly suspected from subsequent ultrasonographic changes of the thyroid and cervical lymph nodes, and the patients underwent reexamination by a fineneedle aspiration biopsy. Because the cytological characteristics of MALT lymphoma and Hashimoto's thyroiditis are similar, it is not uncommon for the cytological diagnosis of MALT lymphoma to be difficult in patients with Hashimoto's thyroiditis (13). A careful ultrasonographic follow-up is important for determining whether to repeat a fine-needle aspiration biopsy or perform an open biopsy in order to diagnose lymphoma.

Despite the ultrasonographic follow-up before the diagnosis of lymphoma, the lymphoma had spread to the thorax during radiotherapy in one patient (Case 1). She had the largest goiter among all the present patients, and her goiter enlarged rapidly just before the diagnosis of lymphoma. Although a greater tumor size, rapid clinical growth, and presence of aggressive histology lymphoma are reported to be prognostic factors of primary thyroid lymphoma (14), we could not identify the histopathological difference between Case 1 and Cases 2-9. Thyroid MALT lymphoma was diag- nosed before the development of subjective symptoms by an ultrasonographic follow-up in most of the present patients (Cases 2-9). Some cases of primary thyroid lymphoma might have been diagnosed as MALT lymphoma by an ultrasonographic follow-up before the transformation to DLBCL. However, the natural history of MALT lymphoma is obscure. Although rare, some lymphomas may spontaneously regress or remit (15). Thus, the benefit of an early diagnosis and treatment of MALT lymphoma for its prognosis remains to be elucidated.

In conclusion, thyroid MALT lymphoma could be diagnosed early from the emergence of a hypoechoic nodular lesion by an ultrasonographic follow-up of Hashimoto's thyroiditis. The growth pattern of thyroid MALT lymphoma differs among individual patients. The beneficial effect of an ultrasonographic follow-up of Hashimoto's thyroiditis on the prognosis of early diagnosed thyroid MALT lymphoma remains to be elucidated by future investigations.

The authors state that they have no Conflict of Interest (COI).

\section{References}

1. Graff-Baker A, Sosa JA, Roman SA. Primary thyroid lymphoma: a review of recent developments in diagnosis and histology-driven treatment. Curr Opin Oncol 22: 17-22, 2010.

2. Moshynska OV, Saxena A. Clonal relationship between Hashimoto thyroiditis and thyroid lymphoma. J Clin Pathol 61: 438-444, 2008.

3. Matsuzuka F, Miyauchi A, Katayama S, et al. Clinical aspects of primary thyroid lymphoma: diagnosis and treatment based on our experience of 119 cases. Thyroid 3: 93-99, 1993.

4. Derringer GA, Thompson LD, Frommelt RA, Bijwaard KE, Heffess CS, Abbondanzo SL. Malignant lymphoma of the thyroid gland: a clinicopathologic study of 108 cases. Am J Surg Pathol 24: 623-639, 2000.

5. Matsuzuka F, Amino N, Kuma K, Miyauchi A. Serial changes in thyroid ultrasonogram in a patient with Hashimoto's thyroiditis who developed malignant lymphoma. Thyroid 15: 742-743, 2005.

6. Tajiri J. Radioactive iodine therapy for goitrous Hashimoto's thyroiditis. J Clin Endocrinol Metab 91: 4497-4500, 2006.

7. Ota H, Ito Y, Matsuzuka F, et al. Usefulness of ultrasonography for diagnosis of malignant lymphoma of the thyroid. Thyroid 16: 983-987, 2006.

8. Watanabe N, Noh JY, Narimatsu H, et al. Clinicopathological features of 171 cases of primary thyroid lymphoma: a long-term study involving 24553 patients with Hashimoto's disease. Br J Haematol 153: 236-243, 2011.

9. Thieblemont C, Mayer A, Dumontet C, et al. Primary thyroid lymphoma is a heterogeneous disease. J Clin Endocrinol Metab 87: 105-111, 2002.

10. Hyjek E, Isaacson PG. Primary B cell lymphoma of the thyroid and its relationship to Hashimoto's thyroiditis. Hum Pathol 19: 1315-1326, 1988.

11. Walsh S, Lowery AJ, Evoy D, McDermott EW, Prichard RS. Thyroid lymphoma: recent advances in diagnosis and optimal management strategies. Oncologist 18: 994-1003, 2013.

12. Robinson KA, Middleton WD. Ultrasonographic evaluation of the thyroid. Ultrasound Clin 9: 325-337, 2014.

13. Sangalli G, Serio G, Zampatti C, Lomuscio G, Colombo L. Fine needle aspiration cytology of primary lymphoma of the thyroid: a report of 17 cases. Cytopathology 12: 257-263, 2001.

14. Stein SA, Wartofsky L. Primary thyroid lymphoma: a clinical review. J Clin Endocrinol Metab 98: 3131-3138, 2013.

15. de Mast Q, Haverman J, Netten PM, Sinnige HA. Remission of a primary thyroid lymphoma after methotrexate withdrawal. Clin Endocrinol (Oxf) 64: 716-717, 2006.

(C) 2016 The Japanese Society of Internal Medicine http://www.naika.or.jp/imonline/index.html 